\title{
Temperature-dependent photoluminescence of self- assembled (In,Ga)As quantum dots on GaAs (100): carrier redistribution through low-energy continuous states
}

\section{Citation for published version (APA):}

Mano, T., Nötzel, R., Gong, Q., Lippen, van, T., Hamhuis, G. J., Eijkemans, T. J., \& Wolter, J. H. (2005).

Temperature-dependent photoluminescence of self-assembled (In, Ga)As quantum dots on GaAs (100): carrier redistribution through low-energy continuous states. Journal of Applied Physics, 44(9A), 6829-6832.

https://doi.org/10.1143/JJAP.44.6829

DOI:

10.1143/JJAP.44.6829

Document status and date:

Published: 01/01/2005

\section{Document Version:}

Publisher's PDF, also known as Version of Record (includes final page, issue and volume numbers)

\section{Please check the document version of this publication:}

- A submitted manuscript is the version of the article upon submission and before peer-review. There can be important differences between the submitted version and the official published version of record. People interested in the research are advised to contact the author for the final version of the publication, or visit the $\mathrm{DOI}$ to the publisher's website.

- The final author version and the galley proof are versions of the publication after peer review.

- The final published version features the final layout of the paper including the volume, issue and page numbers.

Link to publication

\footnotetext{
General rights

- You may freely distribute the URL identifying the publication in the public portal. follow below link for the End User Agreement:

www.tue.nl/taverne

\section{Take down policy}

If you believe that this document breaches copyright please contact us at:

openaccess@tue.nl

providing details and we will investigate your claim.
}

Copyright and moral rights for the publications made accessible in the public portal are retained by the authors and/or other copyright owners and it is a condition of accessing publications that users recognise and abide by the legal requirements associated with these rights.

- Users may download and print one copy of any publication from the public portal for the purpose of private study or research.

- You may not further distribute the material or use it for any profit-making activity or commercial gain

If the publication is distributed under the terms of Article $25 \mathrm{fa}$ of the Dutch Copyright Act, indicated by the "Taverne" license above, please 


\title{
Temperature-Dependent Photoluminescence of Self-Assembled (In,Ga)As Quantum Dots on GaAs (100): Carrier Redistribution through Low-Energy Continuous States
}

\author{
Takaaki MANO*, Richard NÖTZEL, Qian GONG, Twan V. LIPPEN, Gerrit J. HAMHUIS, \\ Tom J. EIJKEMANS and Joachim H. WOLTER
}

eiTT/COBRA Inter-University Research Institute, Eindhoven University of Technology, 5600MB Eindhoven, The Netherlands

(Received April 15, 2005; revised June 2, 2005; accepted June 2, 2005; published September 8, 2005)

Temperature-dependent photoluminescence (PL) studies of an ensemble of self-assembled (In,Ga)As quantum dots (QDs) on GaAs (100) provide insight into the nature of the continuous states between the wetting layer (WL) and QDs. In addition to the well-known anomalous temperature dependence of the PL peak position and width around $90 \mathrm{~K}$ due to carrier (electron-hole pair) redistribution through the WL, we observe a similar behavior at much lower temperatures around $30 \mathrm{~K}$. This behavior is attributed to carrier redistribution through the low-energy continuous states between the WL and QDs, directly proving their quasi-two-dimensional character. The smaller changes in the PL spectra than the WL-induced ones, however, indicate that the carrier redistribution and, thus, the spatial extent of the continuous states are restricted to a limited area around the QDs. This is also supported by the constant integrated PL intensity in this temperature range due to the absence of nonradiative recombination within these areas. [DOI: 10.1143/JJAP.44.6829]

KEYWORDS: quantum dot, photoluminescence, InGaAs, temperatue dependence

\section{Introduction}

The formation and characterization of (In,Ga)As selfassembled quantum dots (QDs) in the Stranski-Krastanov $(\mathrm{S}-\mathrm{K})$ growth mode have been intensively investigated in the last decade not only for possible device applications, but also for fundamental studies of zero-dimensional (0D) physics. ${ }^{1-5)}$ Ideally, the (In,Ga)As QDs are naturally formed on a thin two-dimensional (2D) wetting layer (WL) due to the $2 \mathrm{D}$ to three-dimensional (3D) growth mode transition, $\left.{ }^{4,5}\right)$ having atomic-like discrete energy states. In reality, however, the electronic structure of self-assembled QDs is more complex due to the intrinsic properties and/or structural imperfections of the combined QD-WL system. Photoluminescence excitation (PLE) measurements of single QDs have demonstrated the existence of background absorption due to a density of continuous states that increases gradually between the QD ground-state and the WL states. ${ }^{6-8)}$ It has been suggested that these continuous states originate from structural imperfections that cause a crossover between the OD-QD and 2D-WL character or band-tail states of the WL. ${ }^{6-8)}$ The continuous states have been theoretically attributed recently to the intrinsic crossing of the free-tobound and bound-to-free QD and WL transitions. ${ }^{9}$ )

In this paper we provide insight into the nature of the continuous states by temperature-dependent photoluminescence (PL) measurements of an ensemble of QDs. In addition to the well-known anomalous redshift of the PL peak energy and the minimum of the peak width around $90 \mathrm{~K}$, which are attributed to carrier (electron-hole pair) redistribution through the $2 \mathrm{D}-\mathrm{WL}$ states, we observe a similar behavior around $30 \mathrm{~K}$. Because tunneling between the QDs can be excluded from the data, this behavior evidences real-space carrier redistribution through the lowenergy continuous states between the QD ground-states and WL states and, hence, directly proves their 2D-character. The smaller changes in the PL peak energy and width than

*Present address: Nanomaterials Laboratory, National Institute for Materials Science, 1-2-1 Sengen, Tsukuba, Ibaraki 305-0047, Japan.

E-mail address: MANO.Takaaki@nims.go.jp the WL induced ones and the absence of any PL from the continuous states, however, suggest that they extend over distances smaller than the carrier diffusion length in the states locally around each QD. This hypothesis is also supported by the constant integrated PL intensity of the QDs in this temperature range, indicating the absence of nonradiative recombination centers within these areas of local carrier redistribution in the combined QD-WL system.

To recall the general model, the anomalous temperature dependence of the PL spectra of an ensemble of QDs, i.e., the larger redshift of the PL peak energy than that of the bulk band-gap energy (referred to as Stokes shift in ref. 10) and the reduction of the peak width, reflect the thermal excitation and redistribution of carriers through channels between the QDs in the presence of ground-state energy fluctuation. ${ }^{11-18)}$ The changes in the PL spectra are roughly classified into three stages. First, at low-temperature, the carriers randomly occupy the QDs with equal probability and the PL spectrum reflects the distribution of the QD ground-state energies of the ensemble. The PL spectrum redshifts with temperature as the bulk band-gap energy does, ${ }^{19)}$ and the peak width stays constant. Second, with increasing temperature, carriers are thermally excited to the channels and then redistributed to other QDs within the ensemble. More carriers are excited from smaller QDs with higher ground-state energies and are, thus, preferentially transferred to larger QDs with lower ground-state energies, resulting in the drastic PL peakenergy redshift and peak width reduction. Finally, with further increase in the temperature, the carriers in the energetically deeper QDs are excited with significant probability and the difference in the excitation probabilities for carriers from the higher- and lower-energy QDs becomes smaller. ${ }^{20)}$ As a result, the PL peak-energy redshift starts approaching the bulk band-gap energy dependence and the peak width increases. If tunneling were the main cause for the carrier redistribution, ${ }^{11,23)}$ a qualitatively different PL spectra behavior would be expected. In the presence of resonant or nonresonant phonon-assisted tunneling, the carriers are already redistributed at the lowest temperature and preferentially occupy low-energy QDs. Hence, with temperature increase a blueshift (or reduced redshift com- 
pared with the bulk band-gap energy shift) of the PL would occur due to thermal reoccupation of the higher-energy QDs. Accordingly, the peak width would continuously increase with temperature.

\section{Experimental}

The sample focused on here was grown by conventional solid-source molecular-beam epitaxy (MBE) on a semiinsulating GaAs (100) substrate with miscut smaller than $0.05^{\circ}$. After growth of a 200-nm-thick GaAs buffer layer at $580^{\circ} \mathrm{C}, 5.2$ monolayer (ML) $(1.46 \mathrm{~nm}) \mathrm{In}_{0.36} \mathrm{Ga}_{0.64}$ As was deposited at $500^{\circ} \mathrm{C}$ for QD formation. After $10 \mathrm{~s}$ growth interruption, a $10 \mathrm{~nm}$ GaAs capping layer was grown on the (In,Ga)As layer before the sample was heated up to $580^{\circ} \mathrm{C}$ for growth of $100 \mathrm{~nm} \mathrm{GaAs}$. Finally, the (In,Ga)As QD layer was again deposited at $500^{\circ} \mathrm{C}$ on top of the GaAs surface. The growth rates of $\mathrm{GaAs}$ and $(\mathrm{In}, \mathrm{Ga}) \mathrm{As}$ were $0.24 \mathrm{ML} / \mathrm{s}$ and $0.37 \mathrm{ML} / \mathrm{s}$ calibrated by high-resolution X-ray diffraction (XRD) of (In,Ga)As/GaAs SL structures. The As 4 beam equivalent pressure (BEP) was maintained at $1 \times 10^{-5}$ Torr. The sample was investigated by atomic force microscopy (AFM) in air for the structural characterization. For the PL and PLE measurements, the sample was cooled down in a continuous-flow helium cryostat with a the sample temperature accuracy of $\pm 0.5 \mathrm{~K}$. For the PL measurements, the $532 \mathrm{~nm}$ line of a Nd-YAG laser was used for excitation with a power density of $\sim 0.4 \mathrm{~W} / \mathrm{cm}^{2}$. The PL signal was dispersed by a single monochromator and recorded by a cooled (In,Ga)As charge-coupled device (CCD) camera. For the PLE measurement, a Ti:sapphire laser was used for excitation with a power density of $\sim 5 \mathrm{~kW} / \mathrm{cm}^{2}$. The PLE signal was dispersed by a triple monochromator and recorded by the same $\mathrm{CCD}$ camera.

\section{Results and Discussion}

Figure 1 shows the AFM image of the QDs formed on the surface in the $\mathrm{S}-\mathrm{K}$ growth mode. The QDs are randomly distributed on the sample surface with an average diameter, height, and density of $\sim 35 \mathrm{~nm}, \sim 3.5 \mathrm{~nm}$, and $\sim 3 \times 10^{10}$ $\mathrm{cm}^{-2}$, respectively. Under the PL excitation of these QDs with the power density of $0.4 \mathrm{~W} / \mathrm{cm}^{2}$, we estimate that the

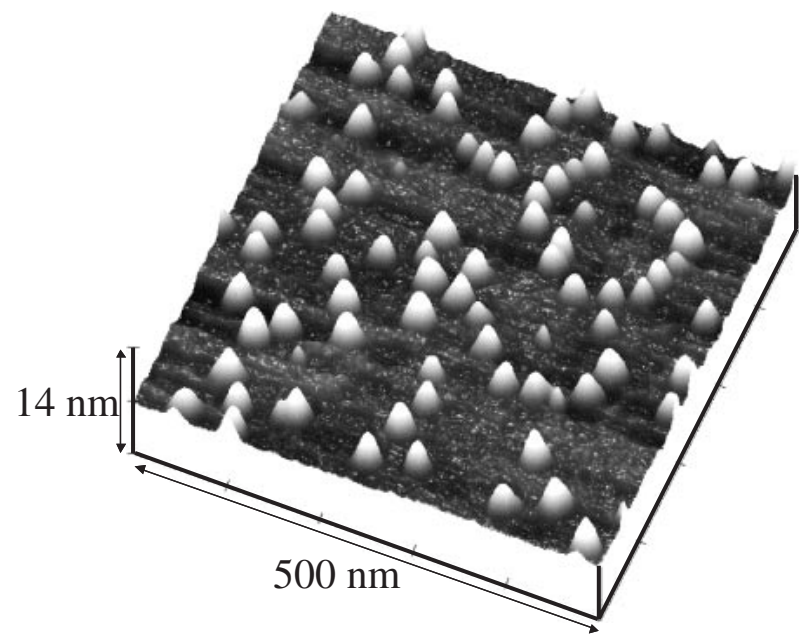

Fig. 1. AFM image of the $1.46 \mathrm{~nm}$ (In,Ga)As QDs on GaAs (100). The scan field is $500 \times 500 \mathrm{~nm}^{2}$

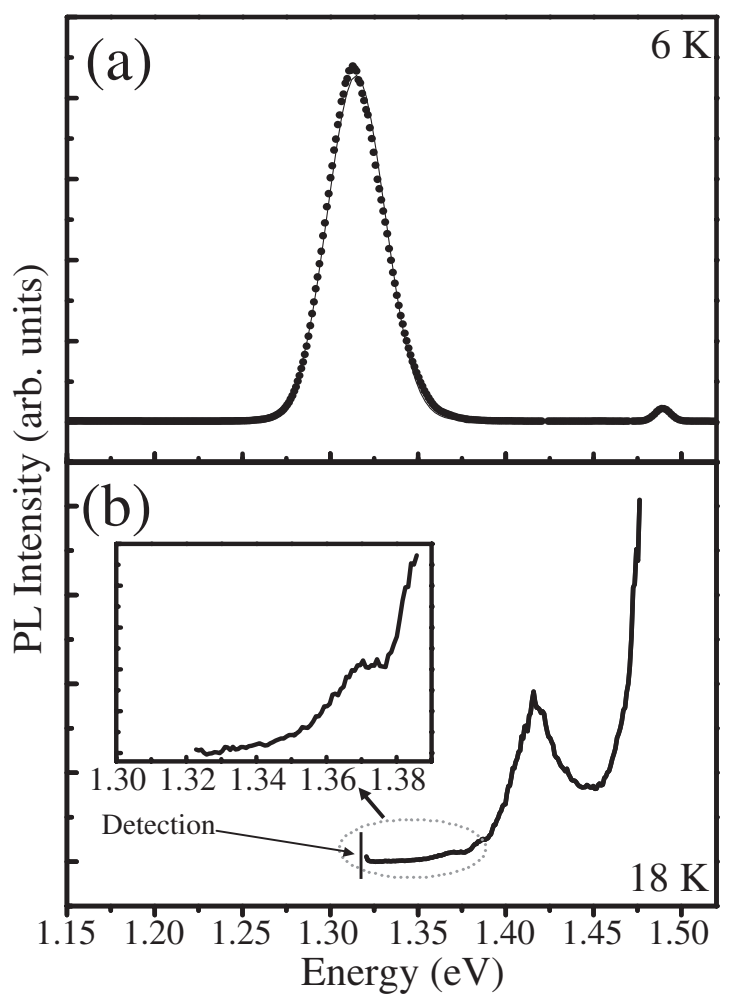

Fig. 2. (a) PL spectrum taken at $6.0 \mathrm{~K}$ from the $1.46 \mathrm{~nm}$ (In,Ga)As QDs on GaAs (100) (filled circles) and the fitting curve using a single Gaussian function (solid line). (b) PLE spectrum of the $1.46 \mathrm{~nm}$ (In,Ga)As QDs on GaAs (100) with the detection energy set at $1.32 \mathrm{eV}$. The inset shows the plot in the low-energy range with an enlarged vertical scale.

average number of carriers in each QD is less than 0.1 , assuming the typical carrier lifetime. ${ }^{21,22)}$ In this low-power excitation condition, the state-filling effect might be negligible. $^{21)}$ Figure 2(a) shows a typical PL spectrum of the buried (In,Ga)As QDs measured at low-temperature. The PL spectrum (full circles) is plotted together with a Gaussian fitting curve (solid line). The PL line centered at $1.32 \mathrm{eV}$ $(943 \mathrm{~nm})$ with a peak width of $32.5 \mathrm{meV}$ corresponds to the emission from the (In,Ga)As QD ensemble. The broadening of the PL line is attributed to the fluctuations in the QD size, shape, and In composition within the measured ensemble containing $10^{8}-10^{9}$ QDs (the diameter of the laser spot is around $0.5 \mathrm{~mm}$ ). The PL line is well fitted by the single Gaussian function. The fitting remains accurate for measurement temperatures below $150 \mathrm{~K}$, indicating only groundstate emissions from the QDs. The weak emission line at $1.49 \mathrm{eV}$ is related to carbon impurities in the GaAs bulk layer. The PLE measurements provide additional information on the electronic structure. Figure 2(b) shows the PLE spectrum with the detection energy set at $1.32 \mathrm{eV}$ at lowtemperature. The clear absorbtion peak at $1.42 \mathrm{eV}$ is related to the WL states. It maintains a constant energy when the detection energy is varied within the QD emission energy range. The increase in absorption above $1.48 \mathrm{eV}$ is due to the GaAs bulk (and/or the WL light hole states ${ }^{24)}$ ). In addition, a continuous absorption tail is visible below $1.35 \mathrm{eV}$, almost approaching the QD ground-state energy [see the inset of Fig. 2(b)]. As has been discussed in previous reports, ${ }^{6-8)}$ this absorption tail is attributed to the continuous states between the WL and QDs. 


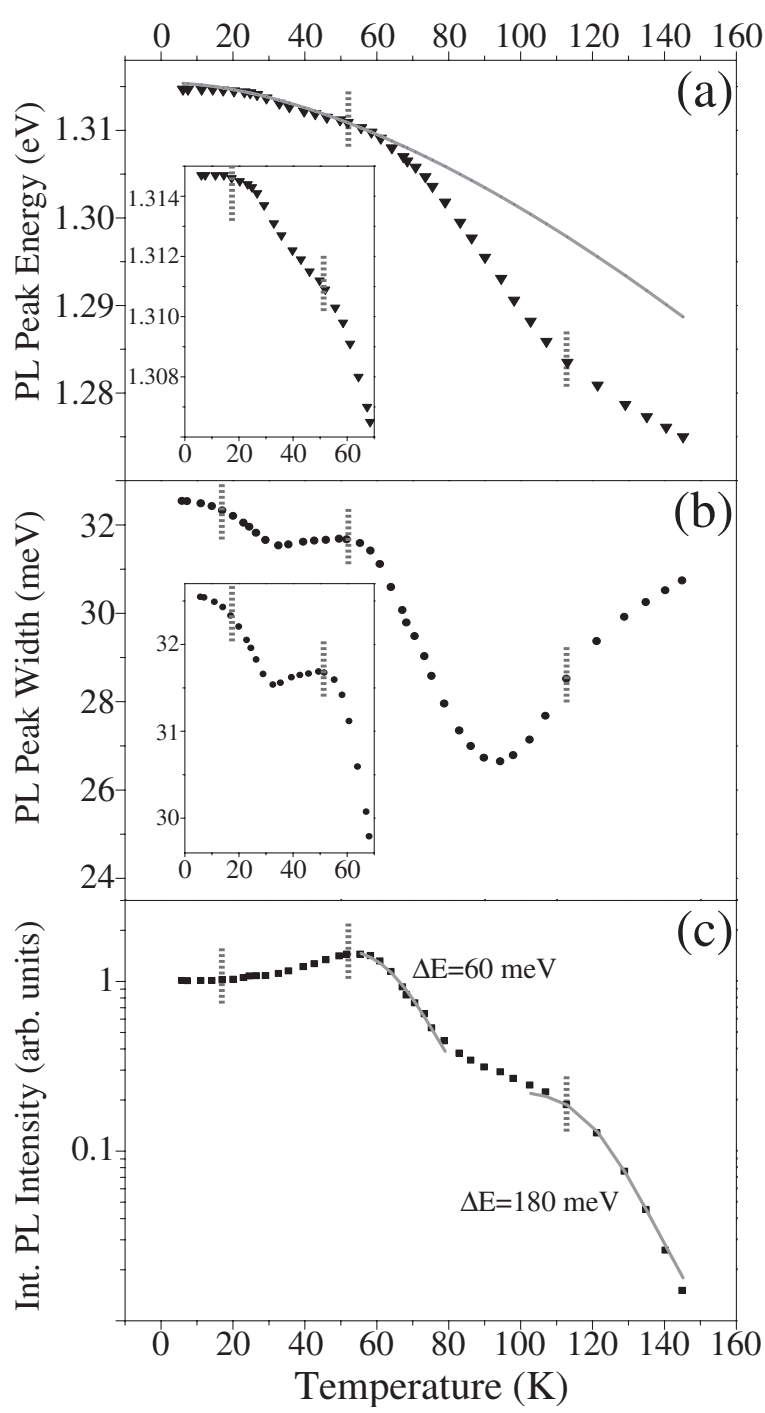

Fig. 3. (a) PL peak position, (b) peak width, and (c) integrated intensity as functions of temperature of the (In,Ga)As QDs. The insets in (a) and (b) show the plots in the low-temperature range with an enlarged vertical scale. The solid line in (a) shows the $\operatorname{In}_{0.36} \mathrm{Ga}_{0.64}$ As bulk band-gap temperature dependence. ${ }^{19)}$ The two solid lines in (c) show the fitting curves in each temperature range. ${ }^{25)}$

Figure 3 shows the temperature dependence of (a) the PL peak energy together with the $\operatorname{In}_{0.36} \mathrm{Ga}_{0.64} \mathrm{As}$ bulk band-gap energy change, ${ }^{19)}$ (b) the peak width, and (c) the integrated intensity of the emissions from the (In,Ga)As QDs, which are determined by the single Gaussian curve fitting of each PL spectrum. The insets of Figs. 3(a) and 3(b) depict the plots in the low-temperature range with an enlarged vertical scale. Compared to the bulk band-gap energy change [solid line in Fig. 3(a)], the PL peak position exhibits two distinct regions of anomalous increase in the redshift around 30 and $110 \mathrm{~K}$, which coincide with characteristic minima of the PL peak width. This clearly reveals a redistribution of carriers between the QDs through distinct sets of continuous energy states.

The drastic redshift of the PL peak energy and pronounced minimum of the peak width between 50 and $110 \mathrm{~K}$ in Figs. 3(a) and 3(b) are assigned to carrier excitation and redistribution through the $2 \mathrm{D}-\mathrm{WL}$ states, connecting a large number of QDs. This is in agreement with previous reports. ${ }^{11,12,14-18)}$ The simultaneous reduction of the inte- grated PL intensity [Fig. 3(c)] is attributed to the activated carrier transport to nonradiative recombination centers in the WL. ${ }^{17)}$ Around $90 \mathrm{~K}$, however, this reduction of the integrated intensity becomes less strong, most likely due to a limited number of nonradiative recombination centers in the WL which get saturated as the number of excited carriers increases. At the same time, the peak energy redshift starts approaching the bulk band-gap energy change, which is accompanied by a broadening of the peak width. This indicates that the excitation probabilities of carriers from the different QDs to the WL become equal. Above $110 \mathrm{~K}$, the integrated PL intensity once again decreases strongly. We believe that carriers are now also efficiently excited into the GaAs bulk and can, thus, escape from the (In,Ga)As QDs and WL. The activation energies $(\Delta E)$ estimated from a simple fitting procedure ${ }^{25)}$ support these assumptions [see the solid lines in Fig. 3(c)]. The activation energies around 65 and $130 \mathrm{~K}$ are $\sim 60$ and $\sim 180 \mathrm{meV}$, respectively. These are roughly consistent with the energy separations of the QD PL peak (at $1.32 \mathrm{eV}$ ) and the onsets of the WL absorption (at $\sim 1.38 \mathrm{eV}$ ) and the GaAs bulk absorption (at $\sim 1.48 \mathrm{eV}$ ) determined from the PLE measurements. This distinction between thermal excitation to the WL and GaAs bulk was not clearly revealed in previous studies. ${ }^{11-18)}$ This might be due to the structurally better defined and wider WL for (In,Ga)As compared to than for InAs QDs ${ }^{26)}$ with welldefined WL and GaAs transition energy separation. Indeed, we were not able to clearly distinguish between thermal excitation to the WL and GaAs bulk for our InAs QD samples studied for comparison.

In addition to the drastic changes induced by the WL and GaAs, a similar anomalous behavior of the PL spectrum is observed in the temperature range between 17 and $52 \mathrm{~K}$, where carriers are unlikely to be excited to the WL. The anomalous redshift of the PL peak position and the clear minimum of the peak width perfectly coincide with the thermal excitation and redistribution model mentioned above. This confirms the presence of additional channels for thermally excited carrier redistribution within the ensemble of QDs, which lie in energy between the WL and QD ground-states. Their density of states is associated with the low-energy absorption tail observed in the PLE measurement [Fig. 2(b)]. ${ }^{6-8)}$ The integrated PL intensity is constant or rather increases in this temperature range, indicating the absence of nonradiative recombination centers in the channels and a higher PL efficiency (longer radiative lifetime ${ }^{23)}$ ) of the low-energy QDs preferentially occupied during the carrier redistribution. The detailed analysis of the temperature-dependent PL peak position and width in this temperature range provides important insight into the properties of these channels. The enhanced redshift and reduction (minimum) of the peak width clearly exhibit onsets or inflection points at $31 \mathrm{~K}$, as shown in the insets of Figs. 3(a) and 3(b). This confirms that the density of the continuous states increases above the QD ground-state energy leading to a behavior like thermal activation over an energy gap. If there were already a significant density of states at the QD ground-state energy, the changes in the PL spectra would be expected to be more continuous without inflection points. In this case also the carriers could efficiently redistribute already at the lowest temperature 
leading to a different PL behavior, i.e., blueshift and broadening. From these results, however, it cannot be concluded whether or not the density of the continuous states approaches zero above the QD ground-state energy.

Most important, the observation of thermally activated carrier redistribution through the continuous states directly proves their quasi-2D extension in real-space. The rather small peak width reduction of only $1 \mathrm{meV}$ compared to that related to the WL of $5 \mathrm{meV}$, however, suggests that the carriers excited to the continuous states are only locally redistributed among the QDs, indicating that the states extend only over a limited area around the QDs. Moreover, the fact that PL from the continuous states is never observed in our experiments and in previous reports indicates that the spatial extent of the continuous states is smaller than the carrier diffusion length within those states. ${ }^{6-8)}$ This local extent is also supported by the rather constant integrated PL intensity. Within these limited areas the carriers do not encounter nonradiative recombination centers.

We have investigated a large series of other (In,Ga)As and InAs QD samples. We always found a qualitatively similar anomalous behavior of the PL peak position and width due to carrier redistribution through the low-energy continuous states for QD densities of $1 \times 10^{10}-1 \times 10^{11} \mathrm{~cm}^{-2}$, independent of In composition, and other parameters such as growth temperature and growth rate. Of course, above a certain QD distance and confinement energy the anomalous behaviors should not be observed due to suppressed carrier redistribution even through the WL. On the other hand, for very dense InAs QD ensembles $\left(\sim 6 \times 10^{11} \mathrm{~cm}^{-2}\right)$, the observed PL peak redshift and peak width reduction in the low-temperature range are even larger, starting from the lowest measurement temperature of $5 \mathrm{~K}$. No inflection points are visible. This indicates stronger spatial overlap of the continuous states for high-density QD and their extension in energy down to the QD ground-state energy, which was also suggested previously, ${ }^{7}$ and might be due to structural effects such as overlapping strain fields. The WL plus GaAsinduced changes of the PL at higher-temperatures remain unaffected by the QD density.

\section{Conclusion}

We have studied the temperature-dependent photoluminescence (PL) of an ensemble of (In,Ga)As quantum dots (QDs) on GaAs (100) grown by molecular-beam epitaxy (MBE). The detailed analysis of the changes in the PL peak energy, peak width, and integrated intensity evidences thermally activated carrier redistribution around $30 \mathrm{~K}$ in addition to the well-known wetting-layer (WL)-related carrier redistribution around $90 \mathrm{~K}$. This behavior proves the quasi-two-dimensional (2D) character of the low-energy continuous states between the WL and QDs. The rather small changes in the PL spectra, however, indicate that the carrier redistribution and, thus, the spatial extent of the continuous states are restricted to a limited area around the QDs without a decrease in the integrated PL intensity due to nonradiative recombination. Similar results from a large set of (In, Ga)As and InAs QD structures suggest that the quasi2D-character of the continuous states is intrinsic to selfassembled QDs grown in the Stranski-Krastanov mode.

1) Y. Arakawa and H. Sakaki: Appl. Phys. Lett. 40 (1982) 939.

2) H. Sakaki: Jpn. J. Appl. Phys. 28 (1989) L314.

3) C. W. Snyder, B. G. Orr, D. Kessler and L. M. Sander: Phys. Rev. Lett. 66 (1991) 3032.

4) D. Leonard, M. Krishnamurthy, C. M. Reaves, S. P. Denbaars and P. M. Petroff: Appl. Phys. Lett. 63 (1993) 3203.

5) Y. Nabetani, T. Ishikawa, S. Noda and A. Sasaki: J. Appl. Phys. 76 (1994) 347.

6) Y. Toda, O. Moriwaki, M. Nishioka and Y. Arakawa: Phys. Rev. Lett. 82 (1999) 4114.

7) C. Kammerer, G. Cassabois, C. Voisin, C. Delalande, Ph. Roussignol and J. M. Gèrard: Phys. Rev. Lett. 87 (2001) 207401.

8) G. Cassabois, C. Kammerer, R. Sopracase, C. Voisin, D. Delalamde, Ph. Roussignol and J. M. Gèrard: J. Appl. Phys. 91 (2002) 5489.

9) A. Vasanelli, R. Ferreira and G. Bastard: Phys. Rev. Lett. 89 (2002) 216804.

10) A. Patanè, A. Levin, A. Polimeni, L. Eaves, P. C. Main, M. Henini and G. Hill: Phys. Rev. B 62 (2000) 11084.

11) D. I. Lubyshev, P. P. González-Borrero, E. Marega, Jr., E. Petitprez, N. La Scala, Jr. and P. Basmaji: Appl. Phys. Lett. 68 (1996) 205.

12) L. Brusaferri, S. Sanguinetti, E. Grilli, M. Guzzi, A. Bignazzi, F. Bogani, L. Carraresi, M. Colocci, A. Bosacchi, P. Frigeri and S. Franchi: Appl. Phys. Lett. 69 (1996) 3354.

13) Y. H. Wu, K. Arai and T. Yao: Phys. Rev. B 53 (1996) R10485.

14) Z. Y. Xu, Z. D. Lu, X. P. Yang, Z. L. Yuan, B. Z. Zheng, J. Z. Xu, W. K. Ge, Y. Wang, J. Wang and L. L. Chang: Phys. Rev. B 54 (1996) 11528.

15) H. Lee, W. Yang and P. C. Sercel: Phys. Rev. B 55 (1997) 9757.

16) A. Polimeni, A. Patanè, M. Henini, L. Eaves and P. C. Main: Phys. Rev. B 59 (1999) 5064.

17) S. Sanguinetti, M. Henini, M. Grassi Alessi, M. Capizzi, P. Frigeri and S. Franchi: Phys. Rev. B 60 (1999) 8276.

18) C. Lobo, N. Perret, D. Morris, J. Zou, D. J. H. Cockayne, M. B. Johnston, M. Gal and R. Leon: Phys. Rev. B 62 (2000) 2737.

19) Y. P. Varshni: Physica (Amsterdam) 34 (1967) 149.

20) With increasing temperature $(T)$, the thermal excitation probabilities $\left(\propto \exp \left(-\Delta E / k_{\mathrm{b}} T\right)\right)$ of carriers from QDs with varying ground-state energies become rather equal. $\Delta E$ is the activation energy and $k_{\mathrm{b}}$ is the Boltzmann constant.

21) S. Raymond, S. Fafard, P. J. Poole, A. Wojs, P. Hawrylak, S. Charbonneau, D. Leonard, R. Leon, P. M. Petroff and J. L. Merz: Phys. Rev. B 54 (1996) 11548.

22) K. Kamath, N. Chervela, K. K. Linder, T. Sosnowski, H.-T. Jiang, T. Norris, J. Singh and P. Bhattacharya: Appl. Phys. Lett. 71 (1997) 927.

23) Yu. I. Mazur, X. Wang, Z. M. Wang, G. J. Salamo, M. Xiao and H. Kissel: Appl. Phys. Lett. 81 (2002) 2469.

24) R. Heitz, M. Veit, N. N. Ledentsov, A. Hoffmann, D. Bimberg, V. M. Ustinov, P. S. Kop'ev and Zh. I. Alferov: Phys. Rev. B 56 (1997) 10435.

25) The integrated PL intensity is fitted by $I=I_{0} /[1+C\{\exp (-\Delta E /$ $\left.\left.\left.k_{\mathrm{b}} T\right)\right\}\right]$. $I_{0}$ and $C$ are fitting parameters.

26) The $2 \mathrm{D}$ to $3 \mathrm{D}$ growth mode transition is observed after the supply of $1.3-1.4 \mathrm{~nm}(\mathrm{In}, \mathrm{Ga}) \mathrm{As}$, which is three times thicker than that for InAs $\left.(0.4-0.5 \mathrm{~nm}){ }^{5}\right)$ 